\title{
A case of functioning gonadotroph adenoma in a reproductive aged woman
}

\author{
Mana Hirano ${ }^{1)}$, Osamu Wada-Hiraike ${ }^{1)}$, Yuichiro Miyamamoto ${ }^{1)}$, Shozo Yamada ${ }^{2)}$, Tomoyuki Fujii ${ }^{1)}$ and \\ Yutaka Osuga ${ }^{1)}$
}

1) Department of Obstetrics and Gynecology, The University of Tokyo Hospital, Tokyo 1138655, Japan

2) Department of Pituitary and Hypothalamic Surgery, Toranomon Hospital, Tokyo 1058470, Japan

\begin{abstract}
A 34-year-old woman presented our hospital with complaint of irregular menstruation and abnormal uterine bleeding lasting for a month. After her second parturition at the age of 27, her menstrual cycle had been regular, but it suddenly became irregular at the age of 30 . Transvaginal ultrasound revealed the presence of ovarian mass, and the patient underwent diagnostic laparoscopic surgery. Bilateral ovaries temporally shrink after puncture but the size soon resumed. Gonadotropins were almost normal, but estradiol and PRL levels turned out to be elevated, and cabergoline treatment was initiated. After referral to our hospital, we found that the ovaries showed multifollicular appearance. Brain magnetic resonance imaging showed an 18-mm macroadenoma in the suprasellar area. To suppress the secretion of endogenous gonadotropins and estrogen, low-dose estrogen-progestin was prescribed. Surprisingly, the treatment temporarily reduced the size of the ovaries. The patient was referred to a neurosurgeon, and a functioning gonadotroph adenoma was suspected. After the resection of the pituitary tumor, her menstrual cycle became regular, and the size of bilateral ovaries became normal. We also noticed that her ovarian reserve judged by anti-Müllerian hormone had been almost diminished after the surgical treatment, probably reflecting the exhaustion of follicular pool. Women with multifollicular ovaries and elevated estradiol levels may have functioning gonadotroph adenomas, although the level of FSH is relatively normal, and ovarian reserve can be followed by measuring anti-Müllerian hormone.
\end{abstract}

Key words: Functioning gonadotroph adenoma, Multifollicular ovaries, Reproductive aged woman, Hyperprolactinemia

THE CASE of functioning gonadotroph adenomas that produce FSH was first described in 1974 [1]. The disease is usually recognized in middle-aged men who have visual impairment, such as decreased vision, seizures, and headaches resulting from enlarged adenomas [2], and the common clinical characteristics are the hypersecretion of FSH. The diagnosis of functioning gonadotroph adenoma in postmenopausal women can be extremely difficult because women after final menstrual period exhibit elevated serum FSH level and the secretion of FSH by adenomas is indistinguishable. A few cases of functioning gonadotroph adenomas in reproductive aged women have been reported and clinical manifestations of the patients are irregular menstrual cycles or amenorrhea, often with enlarged ovaries [3]. The diagnosis FSHproducing pituitary adenomas in reproductive aged

Submitted Feb. 15, 2019; Accepted Mar. 28, 2019 as EJ19-0066 Released online in J-STAGE as advance publication Apr. 19, 2019 Correspondence to: Osamu Wada-Hiraike, Department of Obstetrics and Gynecology, The University of Tokyo Hospital, 7-3-1 Hongo, Bunkyo-ku, Tokyo, Japan.

E-mail: osamuwh-tky@umin.ac.jp women can also be difficult because FSH level could fluctuate during menstrual cycle, and womens in their 30 s exhibit various levels of estrogen considering that the level of FSH reflects ovarian reserve. Here, we report a case of a reproductive aged woman with normal FSH serum levels who exhibited enlarged multifollicular ovaries.

\section{Case Report}

A 34-year-old woman was referred to our hospital because of her irregular menstrual cycles and abnormal uterine bleeding lasting for a month. Her past clinical history and family history before her partitions were unremarkable. Three years after her second parturition, her menstrual cycle became irregular. Her menstrual cycle became normal after receiving oral contraceptive pills, but she quitted taking oral contraceptive pills due to heavy nausea and vomit attribute to the side effects of oral contraceptive pills. The previous doctor found enlarged multifollicular ovarian tumor by transvaginal ultrasound. Laboratory data were obtained including 


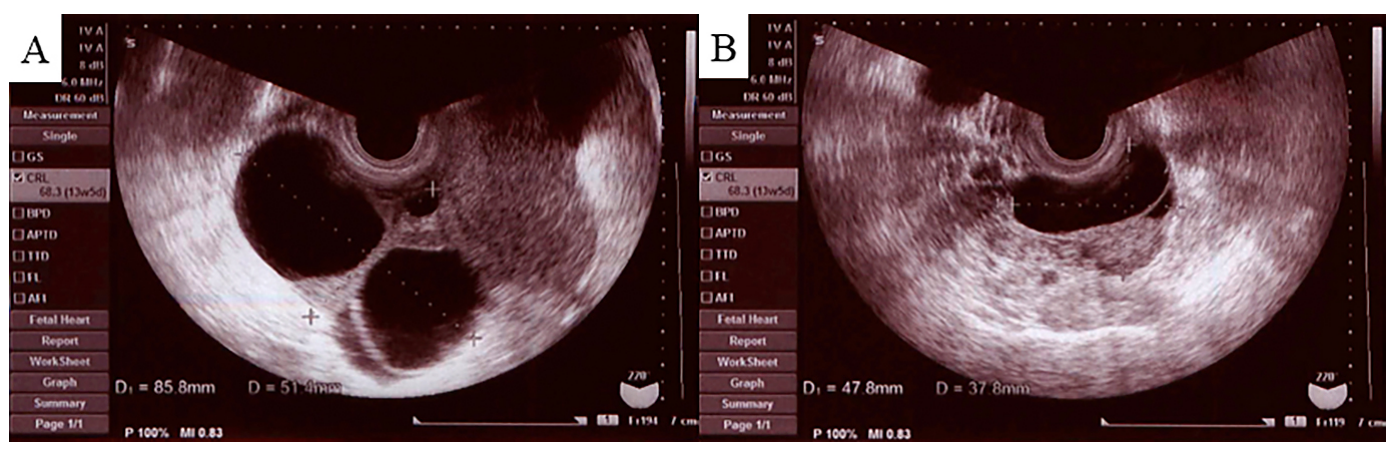

Fig. 1 Transvaginal ultrasound examination showed multifollicular bilateral ovaries.

A. The right ovary was $7 \mathrm{~cm}$ in diameter and polycystic.

B. The left ovary was $5 \mathrm{~cm}$ in diameter.

GnRH test and the data indicated elevated estradiol and PRL levels [FSH (mIU/mL); 7.88 (0 min), 8.13 (30 min), LH (mIU/mL); 0.3 (0 min), 1.61 (30 min), PRL (ng/mL); 71.51 (0 $\mathrm{min}), 31.68$ (60 $\mathrm{min})$ ]. To exclude the possibility of ovarian cancer, the patient underwent diagnostic laparoscopic surgery. Puncture during operation procedure had an influence to reduce the volume of the ovaries, however, the ovaries became enlarged again soon after the operation, and abnormal uterine bleeding started. Although she had no symptom of galactorrhea, cabergoline treatment was initiated to suppress the secretion of PRL. However, her menstrual cycle remained irregular and abnormal uterine bleeding continued. As a result, the patient was referred to our hospital. The laboratory data upon visit of our facility showed that the serum FSH was $12.3 \mathrm{mIU} / \mathrm{mL}$, LH was $0.2 \mathrm{mIU} / \mathrm{mL}$, PRL $4 \mathrm{ng} / \mathrm{mL}$, estradiol $\left(\mathrm{E}_{2}\right)$ was $180.4 \mathrm{pg} / \mathrm{mL}$, progesterone $\left(\mathrm{P}_{4}\right)$ was $2.4 \mathrm{ng} / \mathrm{m}$, and AMH (anti-Müllerian hormone) was $1.01 \mathrm{ng} / \mathrm{mL}$. A transvaginal ultrasound examination revealed a myoma in the uterus, and bilateral ovaries were enlarged, resembling the ovaries under controlled ovarian stimulation in in vitro fertilization program (Fig. 1). Magnetic resonance imaging (MRI) revealed the presence of a pituitary tumor in the suprasellar area (Fig. 2), thus we suspected the possibility of pituitary prolactinoma. Considering that the FSH level was slightly increased and $\mathrm{E}_{2}$ level was also increased, we prescribed low-dose estrogen-progestin (LEP) that contains drospirenone $3 \mathrm{mg}$ and ethinylestradiol 0.02 $\mathrm{mg}$ /day. Cyclic regimen for 16 weeks was continued and LEP temporarily suppressed the pituitary-gonadotropin axis, and ovarian volume decreased; however, the ovarian swelling soon resumed. The patient was referred to a neurosurgeon. In the $500 \mathrm{ng}$ thyrotropin-releasing hormone (TRH) test, serum PRL levels increased from 14 to $44.6 \mathrm{ng} / \mathrm{mL}$, and TSH levels increased from 0.107 to $1.191 \mathrm{mIU} / \mathrm{mL}$ at $30 \mathrm{~min}$ after administration. In the 100 ng gonadotropin-releasing hormone $(\mathrm{GnRH})$ test, serum
FSH was increased from 5.9 to $9.7 \mathrm{mIU} / \mathrm{mL}$, the $\mathrm{LH}$ level was increased from 1.8 to $11.5 \mathrm{mIU} / \mathrm{mL}$ at $30 \mathrm{~min}$ after administration, and the serum FSH level was increased continuously. Taken together, a functioning gonadotroph adenoma was suspected, and the patient underwent resection of the pituitary tumor. Immunohistochemical examination of the resected adenoma showed intense reactivity for FSH and LH (Fig. 3). Finally, her menstrual cycle became regular, and the ovaries became normal in size and AMH was $0.61 \mathrm{ng} / \mathrm{mL}$ after the surgery. Six months later, AMH was below detection level (less than $0.1 \mathrm{ng} / \mathrm{mL}$ ). A functioning gonadotroph adenoma recurred at four years later, so the patient underwent Gamma Knife radiosurgery. At the time of recurrence, her ovaries were normal. She had no symptoms after the surgery and her menstruation remained to be normal.

\section{Discussion}

Functioning gonadotroph adenoma was initially considered to be rare, but recent studies have shown that these tumors account for 20 to $25 \%$ of all pituitary macroadenomas in men $[2,4]$. Histological evidence shows that functioning gonadotroph adenomas occur as frequently in women as they do in men $[5,6]$. The clinical features of functioning gonadotroph adenomas might differ between men and women because the ovaries exhibit multifollicular appearance due to the continuous stimulation by FSH in reproductive aged women, and it can be difficult to diagnose functioning gonadotroph adenomas in postmenopausal women who already have elevated serum concentrations of gonadotropins [7]. Approximately 30 cases functioning gonadotroph adenomas in reproductive-aged women have been reported so far [3, 8]. In most of the reported cases, serum FSH levels ranged from normal to high [9], serum LH levels were decreased, $E_{2}$ levels ranged from normal to high, and 


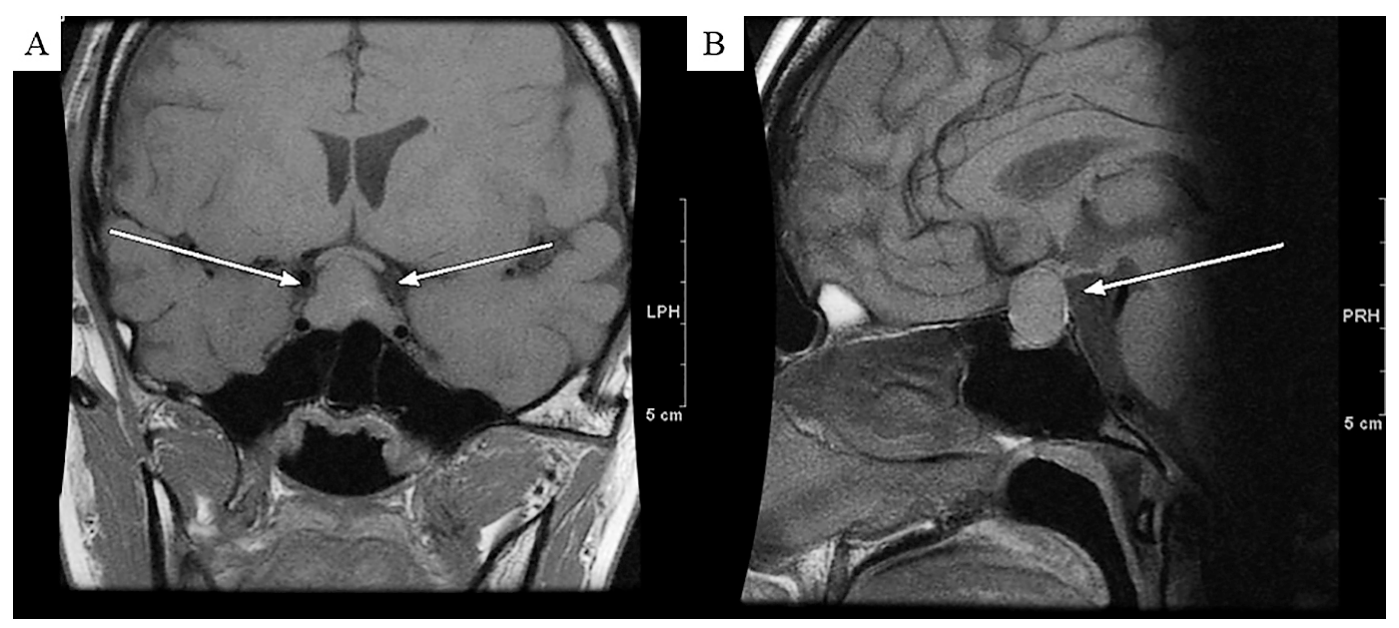

Fig. 2 Brain MRI showed a $1.8 \mathrm{~cm}$ pituitary tumor in the suprasellar area. Arrow head denotes the tumor.

A. Coronal view

B. Sagittal view

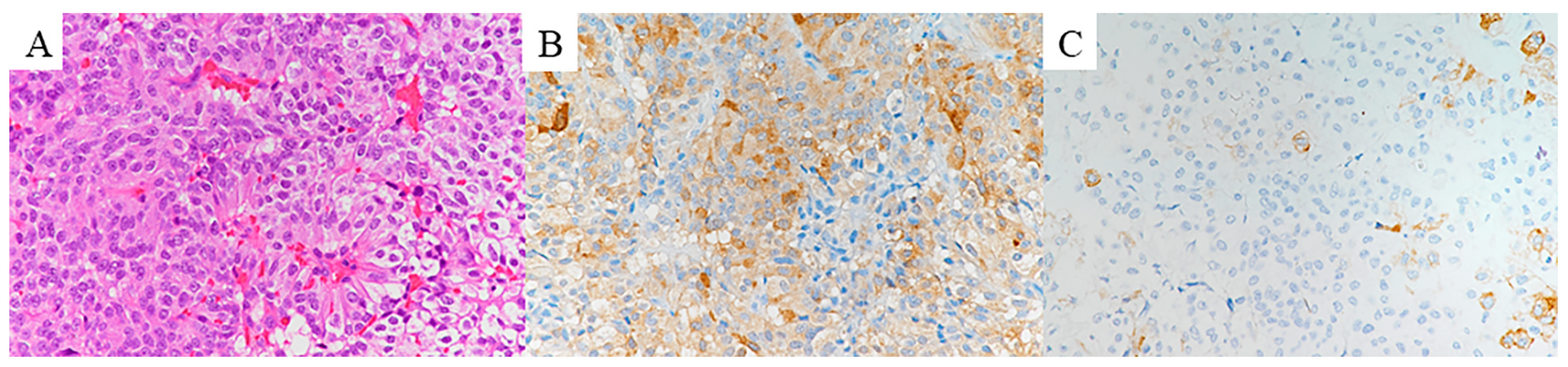

Fig. 3 Pathological findings of the tumor. Immunohistochemistry of the tumor showed positive results for FSH and LH and negative results for PRL, TSH, and ACTH (data not shown).

A. In hematoxylin-eosin staining showed spindle-shaped eosinophilic cells.

B. Over $70 \%$ of the cells were positive for FSH.

C. Approximately, $10-20 \%$ of the cells were positive for $\mathrm{LH}$ (magnified by 40 diameters).

serum PRL levels were elevated. The elevated level of FSH from adenomas leads to the formation of multiple ovarian follicles and increasing $E_{2}$ levels, which results in decreased LH levels and normal FSH levels by an estrogen-induced negative feedback mechanism [10-12]; however, this mechanism is not present with normal FSH and normal $\mathrm{E}_{2}$ levels. A case report showed that the serum FSH bioactivity/immunoactivity ratio of a patient with functioning gonadotroph adenoma was mostly equal to that of two healthy subjects by a granulosa cell aromatase assay, suggesting that other explanations may be necessary for multiple ovarian cysts [13]. An abnormal form of FSH from an adenoma may inhibit pituitary LHreleasing hormone (LHRH) receptors or LHRH secretion from the hypothalamus and lead to decreased LH levels [14]. In perimenopause to postmenopausal women, FSH is elevated due to the decrease of $E_{2}$, thus the ovaries of postmenopausal women with functioning gonadotroph adenomas are often normal in size [8]. In this case, we measured a representative ovarian reserve marker AMH known to be a representative marker for evaluating ovarian reserve, and the changes of AMH during the clinical course of functioning gonadotroph adenoma has not been reported in previous report. This case exhibited decreased AMH level after sphenoidal surgery and the decrement might be attributed to the exhaustion of antral follicles provoked by elevated FSH. We would like to emphasize that this case did not show enlarged ovaries after recurrence of pituitary tumor, and the reason might be hypothesized to the decreased AMH value. Therefore, $\mathrm{AMH}$ can be a good marker for predicting ovarian appearance in cases of reproductive aged women.

The treatment for functioning gonadotroph adenomas is transsphenoidal tumor resection. If a tumor is a microadenoma, then medical treatment is effective in some cases $[15,16]$. 
The present case showed normal FSH levels, almost normal estradiol levels, and elevated PRL with enlarged ovaries. Notably, women with multicystic enlarged ovaries and elevated estradiol and prolactin levels may have functioning gonadotroph adenoma, but the ovaries will not be enlarged in patients with decreased ovarian reserve.

\section{Acknowledgments}

We thank Naoko Inoshita for giving the figures of immunohistochemistry. This work was supported by Ministry of Health, Labour and Welfare.

\section{Disclosure}

None of the authors have any potential conflicts of interest to disclose.

\section{References}

1. Woolf PD, Schenk EA (1974) An FSH-producing pituitary tumor in a patient with hypogonadism. $J$ Clin Endocrinol Metab 38: 561-568.

2. Snyder PJ (1987) Gonadotroph cell pituitary adenomas. Endocrinol Metab Clin North Am 16: 755-764.

3. Halupczok J, Kluba-Szyszka A, Bidzinska-Speichert B, Knychalski B (2015) Ovarian hyperstimulation caused by gonadotroph pituitary adenoma-review. Adv Clin Exp Med 24: 695-703.

4. Beckers A, Stevenaert A, Mashiter K, Hennen G (1985) Follicle-stimulating hormone-secreting pituitary adenomas. J Clin Endocrinol Metab 61: 525-528.

5. Mashiter K, Adams E, Van Noorden S (1981) Secretion of LH, FSH, and PRL shown by cell culture and immunocytochemistry of human functionless pituitary adenomas. Clin Endocrinol (Oxf) 15: 103-112.

6. Kwekkeboom DJ, de Jong FH, Lamberts SW (1989) Gonadotropin release by clinically nonfunctioning and gonadotroph pituitary adenomas in vivo and in vitro: relation to sex and effects of thyrotropin-releasing hormone, gonadotropin-releasing hormone, and bromocriptine. $J$ Clin Endocrinol Metab 68: 1128-1135.

7. Daneshdoost L, Gennarelli TA, Bashey HM, Savino PJ, Sergott RC, et al. (1991) Recognition of gonadotroph adenomas in women. $N$ Engl J Med 324: 589-594.

8. Ntali G, Capatina C, Grossman A, Karavitaki N (2014) Clinical review: functioning gonadotroph adenomas. $J$ Clin Endocrinol Metab 99: 4423-4433.

9. Broughton C, Mears J, Williams A, Lonnen K (2018) A clinically functioning gonadotroph adenoma presenting with abdominal pain, ovarian hyperstimulation and fibromatosis. Endocrinol Diabetes Metab Case Rep doi: 10.1530/EDM-18-0123 [Epub ahead of print].
10. Kihara M, Sugita T, Nagai Y, Saeki N, Tatsuno I, et al. (2006) Ovarian hyperstimulation caused by gonadotroph cell adenoma: a case report and review of the literature. Gynecol Endocrinol 22: 110-113.

11. Shimon I, Rubinek T, Bar-Hava I, Nass D, Hadani M, et al. (2001) Ovarian hyperstimulation without elevated serum estradiol associated with pure follicle-stimulating hormone-secreting pituitary adenoma. J Clin Endocrinol Metab 86: 3635-3640.

12. Djerassi A, Coutifaris C, West VA, Asa SL, Kapoor SC, et al. (1995) Gonadotroph adenoma in a premenopausal woman secreting follicle-stimulating hormone and causing ovarian hyperstimulation. $J$ Clin Endocrinol Metab 80: 591-594.

13. Kajitani T, Liu S, Maruyama T, Uchida H, Sakurai R, et al. (2008) Analysis of serum FSH bioactivity in a patient with an FSH-secreting pituitary microadenoma and multicystic ovaries: a case report. Hum Reprod 23: 435-439.

14. Pentz-Vidovic I, Skoric T, Grubisic G, Korsic M, IvicevicBakulic T, et al. (2000) Evolution of clinical symptoms in a young woman with a recurrent gonadotroph adenoma causing ovarian hyperstimulation. Eur J Endocrinol 143: 607-614.

15. Garmes HM, Grassiotto OR, Fernandes YB, Queiroz Lde $\mathrm{S}$, Vassalo J, et al. (2012) A pituitary adenoma secreting follicle-stimulating hormone with ovarian hyperstimulation: treatment using a gonadotropin-releasing hormone antagonist. Fertil Steril 97: 231-234.

16. Karapanou O, Tzanela M, Tamouridis N, Tsagarakis S (2012) Gonadotroph pituitary macroadenoma inducing ovarian hyperstimulation syndrome: successful response to octreotide therapy. Hormones (Athens) 11: 199-202. 